\title{
Neurobiology of dyslexia: a reinterpretation of the data
}

\author{
Franck Ramus ${ }^{1,2}$
}

${ }^{1}$ Laboratoire de Sciences Cognitives et Psycholinguistique (EHESS/CNRS/ENS), 46 rue d'UIm, 75230 Paris Cedex 5, France

${ }^{2}$ Institute of Cognitive Neuroscience, University College London, 17 Queen Square, London WC1N 3AR, UK

\begin{abstract}
Theories of developmental dyslexia differ on how to best interpret the great variety of symptoms (linguistic, sensory and motor) observed in dyslexic individuals. One approach views dyslexia as a specific phonological deficit, which sometimes co-occurs with a more general sensorimotor syndrome. This article on the neurobiology of dyslexia shows that neurobiological data are indeed consistent with this view, explaining both how a specific phonological deficit might arise, and why a sensorimotor syndrome should be significantly associated with it. This new conceptualisation of the aetiology of dyslexia could generalize to other neurodevelopmental disorders, and might further explain heterogeneity within each disorder and comorbidity between disorders.
\end{abstract}

Developmental dyslexia is a mild hereditary neurological disorder that manifests as a persistent difficulty in learning to read in children with otherwise normal intellectual functioning and educational opportunities. Researchers typically attempt to characterize dyslexia at the genetic, neurobiological and cognitive levels of description, and to uncover causal pathways between the different levels.

One notable aspect of dyslexia that puzzles theorists and causes much confusion is the variety of symptoms that are consistently associated with it: problems with reading, of course, but also problems with phonology (the mental representation and processing of speech sounds), sensory difficulties in the visual, auditory and tactile domains, problems with balance and motor control, and more [1,2]. Another puzzle is that dyslexia is frequently comorbid with other neurodevelopmental disorders, such as specific language impairment (SLI), attention deficit hyperactivity disorder (ADHD) or dyspraxia $[3,4]$.

This plurality of symptoms has led to two broad approaches to dyslexia. One has been to concentrate on one particular cognitive symptom thought to reflect the most direct causal explanation: for instance, in the phonological theory of dyslexia [5] (Figure 1a), a specific deficit in the representation and processing of speech sounds is thought to cause difficulty in learning and handling the relationship between letters and speech sounds (grapheme-phoneme correspondences). Within this approach, the other symptoms of dyslexia are

Corresponding author: Franck Ramus (franck.ramus@ens.fr).

Available online 14 October 2004 considered as simple comorbid markers, without causal relationship with the reading disability.

Conversely, the alternative theoretical approach gives a primary explanatory role to the sensory and/or motor symptoms. This approach has led to the formulation of theories of dyslexia tracing the causes of reading disability back to auditory (temporal) processing deficits (via the phonological deficit) [6,7], visual (magnocellular) dysfunction $[8,9]$ and/or motor (cerebellar) dysfunction [10,11]. The culminating point of this approach has been the unification of its different variants under the general magnocellular theory (Figure 1b), in which a generalized dysfunction of cells in the magnocellular pathway affects all sensory modalities and prolongs itself in the posterior parietal cortex and the cerebellum [1]. Uniquely, this theory accounts for reading disability both through auditory-phonological and visual-spatial deficits, and encompasses all known cognitive, sensory and motor manifestations of dyslexia.

However, as I have argued elsewhere [2,12], the magnocellular theory only partly succeeds in explaining the whole dataset. In particular, it fails to explain why the prevalence of sensorimotor dysfunction is so much lower than that of the phonological deficit in the dyslexic population. Even within the subset of dyslexics affected by sensory and/or motor disorders, the causal relationship with the reading impairment is far from clear $[2,13]$. On the basis of a comprehensive review of the literature, I have previously advocated that dyslexia is, in most individuals, explained by a specific phonological deficit; furthermore, a general sensorimotor syndrome occurs more often in the dyslexic than in the general population, but does not by itself play a significant causal role in the aetiology of the reading impairment [2]. This paper reviews the neurobiology of dyslexia and argues that the available data do indeed support this view, by explaining both how a specific phonological deficit might arise and why a sensorimotor syndrome should be significantly associated with it.

\section{Data from anatomical studies}

Post-mortem examination and brain imaging studies have documented many differences between dyslexic and control brains, for example in the left perisylvian cortex, the underlying white matter, the thalamus, the corpus callosum, and the cerebellum (see Refs $[14,15]$ for reviews). In most cases, the functional significance of 
(a) The phonological theory - - -

(b) The magnocellular theory - - -

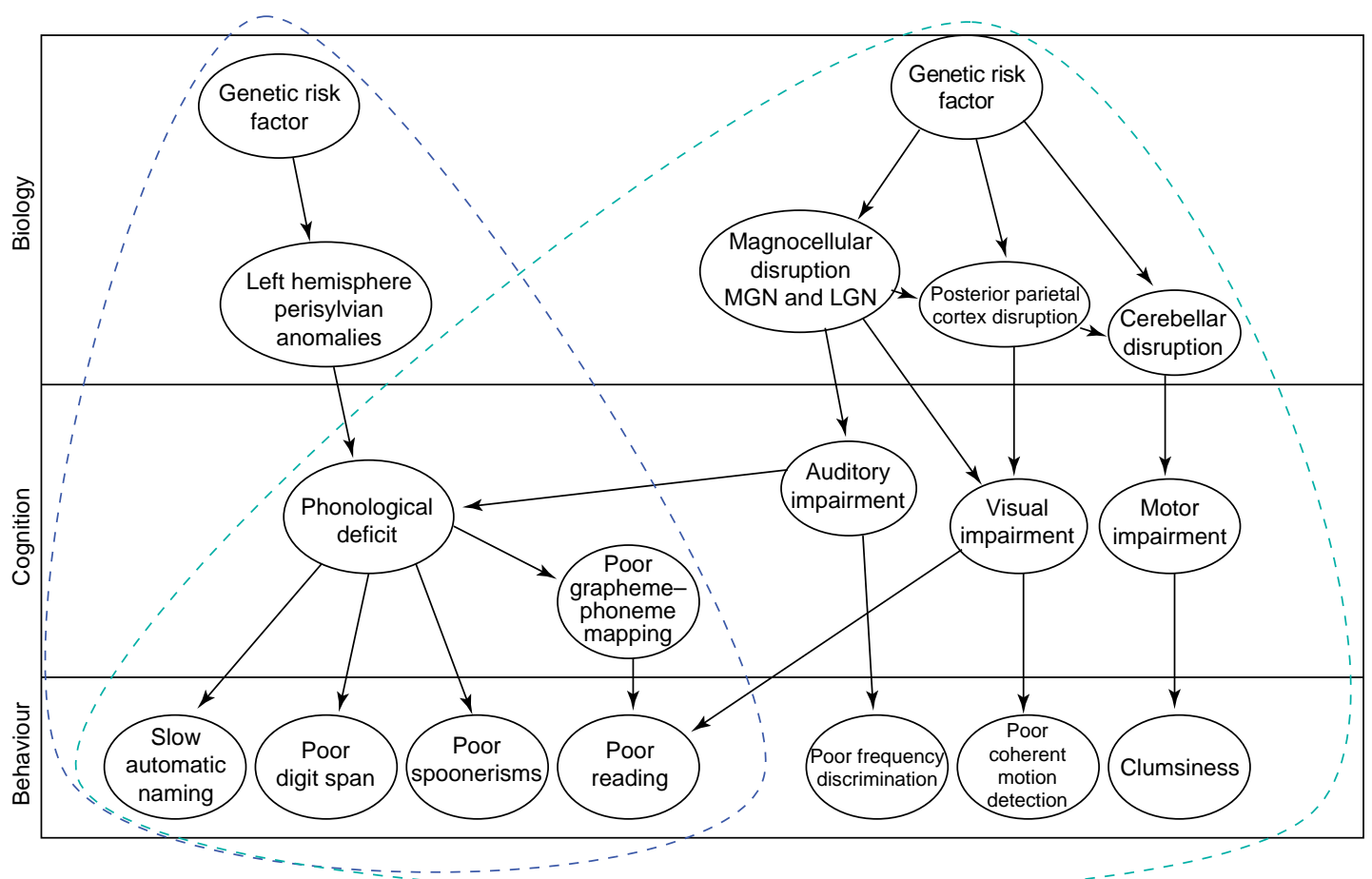

(c) The proposed model

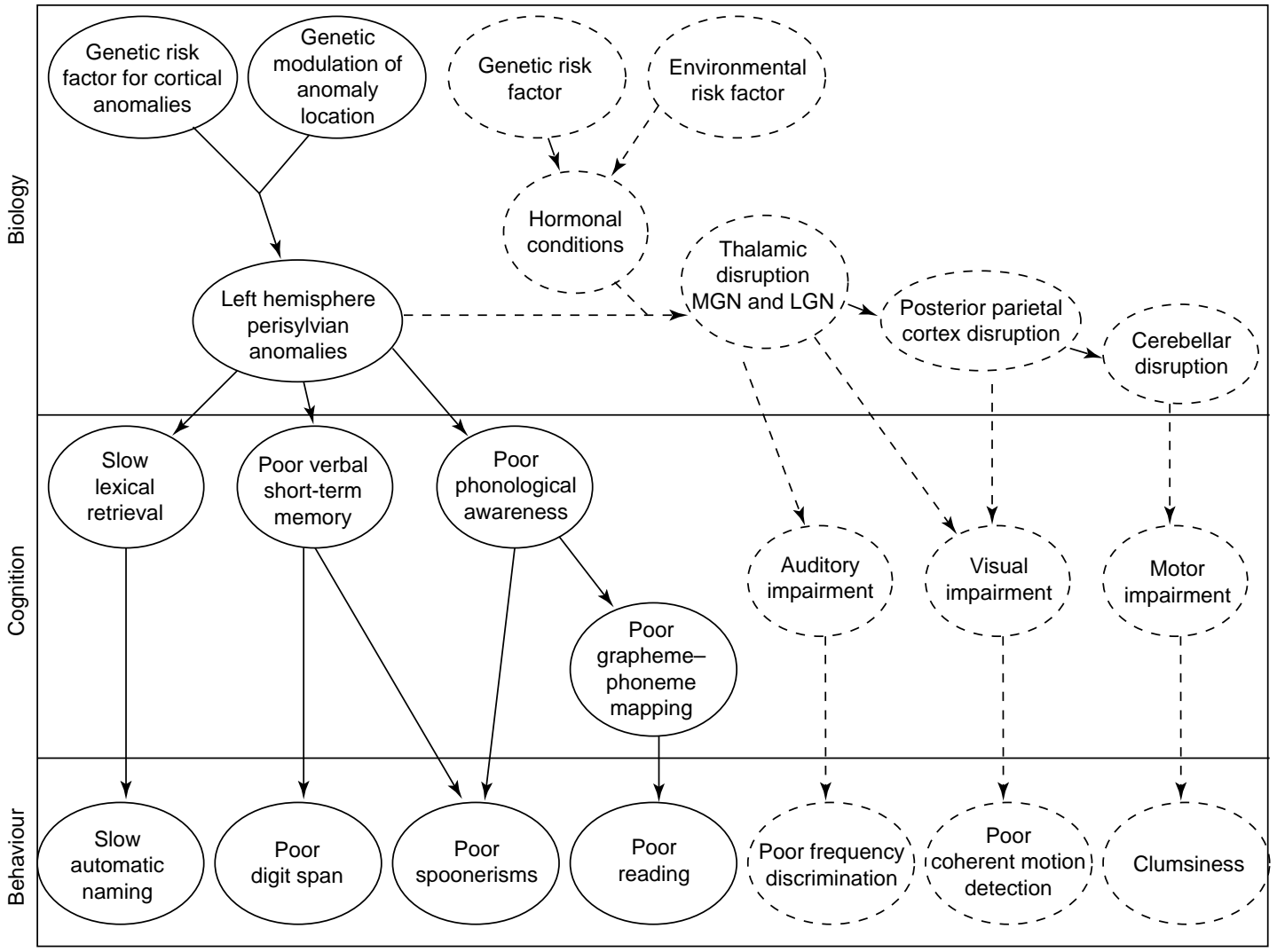

$\overline{\text { TRENDS in Neurosciences }}$

Figure 1. Three causal models of the aetiology of developmental dyslexia. Ovals represent traits at the biological, cognitive and behavioural levels of description; arrows represent causal relationships between traits. Only a subset of all possible behavioural manifestations is represented. (a) Traits and relationships postulated by the phonological theory. (b) Traits and relationships postulated by the magnocellular theory. (c) The proposed model. Solid lines are used for core traits of developmental dyslexia, dashed lines for associated traits that are not necessarily present in each affected individual. Cases of comorbidity with other developmental disorders (e.g. specific language impairment) are not represented. Abbreviations: LGN, lateral geniculate nucleus; MGN, medial geniculate nucleus. 
these brain differences has not been elucidated. It is not even clear which of those differences are specifically relevant to dyslexia, given comorbidity issues. Nevertheless, the functional significance of two types of brain anomaly has been studied in greater detail.

Anomalies of cell migration (molecular layer ectopias and focal microgyri) have been observed by Galaburda and colleagues in the perisylvian cortex of dyslexic brains [16-18], predominantly in the left hemisphere, and with a much greater prevalence than in control brains [19]. Ectopias consist of 50-100 neurons (and glia) that, in the course of neural migration, have missed their target in the cortex and have escaped into the molecular layer through a breach in the external glial limiting membrane, accompanied by mild disorganization of the subjacent cortical layers (Figure 2). Microgyri are more severe disturbances where organization of all layers of the cortex is severely affected. Cytoarchitectonic anomalies have also been observed in the thalamus of dyslexics: in the lateral geniculate nucleus (LGN), the magnocellular layers were more disorganized and contained smaller cell bodies [9]. Similarly, there was a disproportionate number of small neurons in left medial geniculate nucleus (MGN) of dyslexics [20].

It is natural to hypothesize that anomalies in the magnocellular layers of the LGN are the cause of visual deficits, and that anomalies in the MGN are the cause of auditory deficits. Similarly, it is easy to see cortical anomalies in left perisylvian areas as the underlying cause of phonological, and perhaps other, cognitive difficulties.

In this anatomical evidence, one can therefore see direct neurological support for auditory and magnocellular theories of dyslexia. The implicit causal (bottom-up) scenario is that anomalies in the thalamus engender ectopias and microgyri in certain cortical areas to which the thalamus is connected. At the cognitive level, this would translate into the auditory deficit causing a phonological deficit, and into the basic visual deficit causing visual-spatial attentional problems, as prescribed

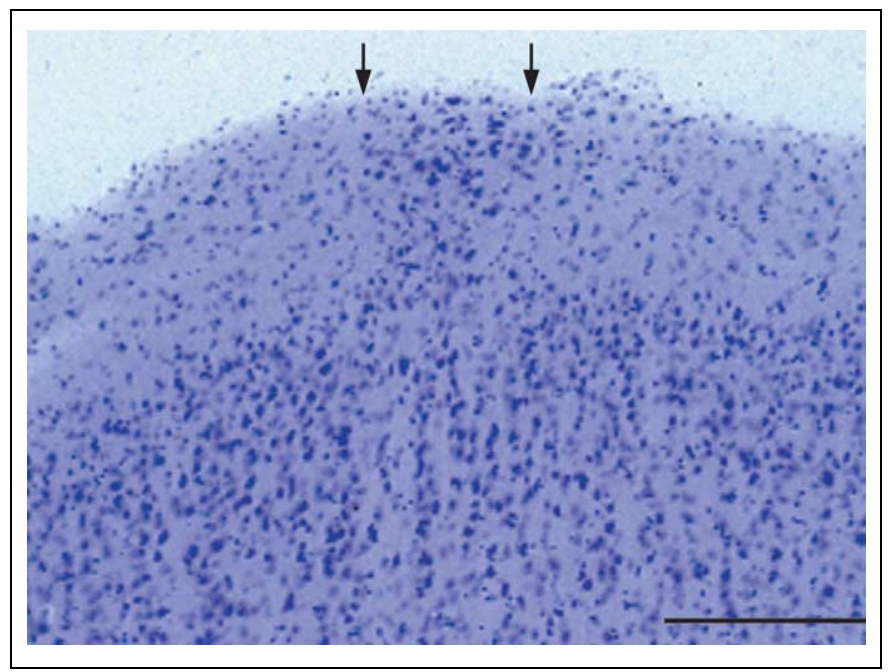

Figure 2. A molecular layer ectopia in a dyslexic subject. Neurons and glia have escaped into the molecular layer of the cortex, through a breach in the external glial limiting membrane, to form an ectopia (between the two arrows). Scale bar, $250 \mu \mathrm{m}$. Micrograph kindly provided by Glenn D. Rosen. by the magnocellular theory. However, this scenario might well be incorrect [21]. Indeed, Galaburda and colleagues have found that, at least in animal models, the causal direction seems to be the opposite (top-down) - that is, the cortical anomalies engender the thalamic anomalies.

\section{More insights from animal models}

In rats, one can surgically induce ectopias and microgyri by poking a hole in the external glial limiting membrane of the developing cortex during late neocortical neuronal migration. There are also strains of mutant mice that spontaneously develop similar malformations. Investigation of these animal models has led to several important findings.

First, newborn rats with microgyri in the frontal, parietal or occipital cortex subsequently develop anomalies in the MGN: they have more small and fewer large neurons than rats receiving sham lesions, a disruption similar to that found in the MGN of dyslexics [22,23]. This suggests that the direction of causation is indeed topdown, from the cortex to sensory relays in the thalamus. Furthermore, rats with this type of abnormal MGN performed less well in an auditory discrimination task [22-24], which confirms that the observed disruption in the MGN has an impact on auditory capacities. Similar auditory disorders are found in mice with ectopias [25], suggesting that this top-down scenario might also occur when cortical malformations have a genetic origin. Extrapolated to dyslexia, these findings suggest that the neural basis of the phonological deficit could be primary, whereas the neural basis for sensory impairments would be secondary.

Another interesting aspect uncovered in these studies is that only male rats and mice with microgyri or ectopias were initially found to have impaired auditory function $[26,27]$. Female rats showed normal auditory performance and did not show a similar anatomical disruption of the MGN, even though they presented with microgyri as severe as those in males [22]. It was then found that this sex difference had a hormonal basis; indeed, female rats that were androgenized by injection of testosterone during gestation showed MGN disruption and impaired auditory function in the same way as males [28]. This therefore suggests that the neural basis for the phonological deficit can occur either with or without the secondary sensory impairments, depending on whether certain hormonal conditions are met.

Finally, the cortical anomalies themselves seem to have an impact on cognitive function: mice and rats with spontaneous or induced ectopias and microgyri exhibit a variety of learning deficits [29-32], including problems with working memory [33-35]. Furthermore, the location of the cortical disruption influences the specific type of learning deficit exhibited by the animal $[36,37]$, but not the likelihood of further thalamic disruption and sensory impairment [25]. This suggests that the location of cortical abnormalities will be crucial to the nature of the cognitive deficits observed in dyslexia, whereas sensory impairments can be expected to arise regardless of the cortical locus and specific type of cognitive deficit. 


\section{Scaling up to dyslexia}

Although the data already reviewed here are not sufficiently constraining to specify a single neurobiological model of dyslexia, they seem most compatible with one based on the following hypotheses (Figure 1c):

- Genetically driven focal cortical abnormalities such as ectopias and microgyri, in specific areas of left perisylvian cortex involved in phonological representations and processing, are the primary cause of dyslexia (Figure 3). This is consistent with: (i) anatomical studies of dyslexic brains showing loci of cortical abnormalities; (ii) functional brain imaging studies showing that the very same areas are involved in phonological processing, and show abnormal activation in dyslexics; (iii) mouse models with ectopias, as already discussed; and (iv) recent findings that the dyslexia susceptibility gene DYX1C1 is involved in neural migration, and that the deletion found in a dyslexic family disrupts its function (Y. Wang et al., unpublished).

- Under certain hormonal conditions (which might or might not reduce to elevated levels of fœtal testosterone), the disruption propagates to the thalamus, provoking additional (and optional) sensory impairments. This is

(a)

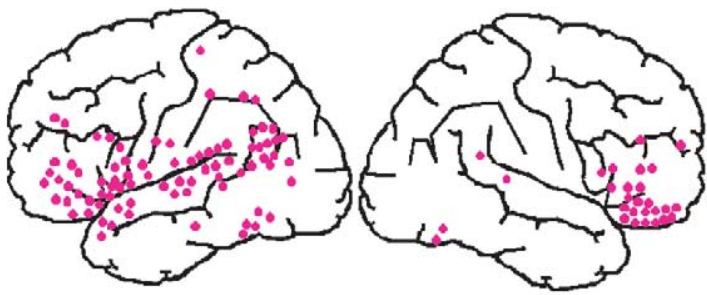

(b)

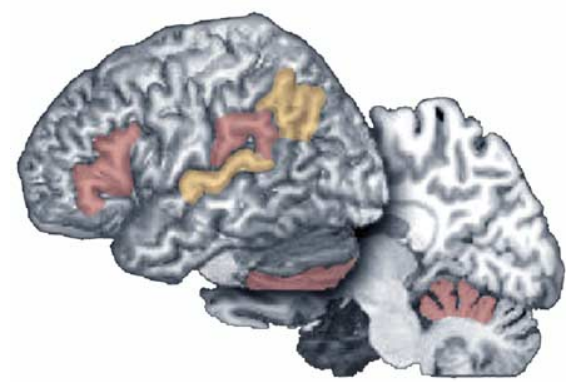

(c)
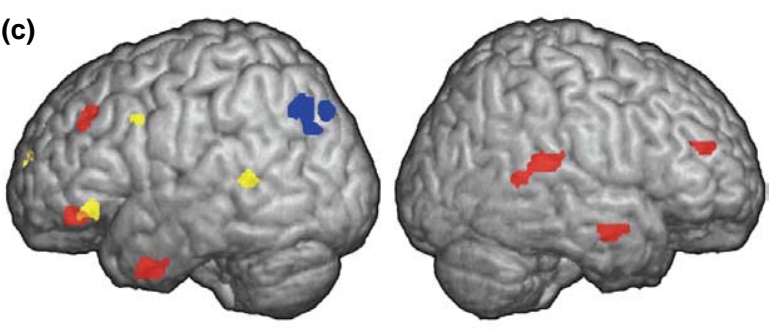

Figure 3. Neurobiology of developmental dyslexia. (a) Overall distribution of cortical ectopias observed across different dyslexic subjects (kindly provided by Glenn D. Rosen). (b) Brain areas activated in oral language tasks and exhibiting structural differences between dyslexics and controls. Areas in orange are supported by one published study, areas in red by more than one. Reproduced, with permission of Sage Publications, Inc., from Ref. [15]. (c) Brain areas activated during performance of the main phonological skills impaired in dyslexia: phonological awareness (yellow), rapid serial naming (red) and verbal short-term memory (blue). Reproduced, with permission, from Ref. [39]. consistent with rat and mouse models and the fact that sensory disorders are present in some, but not all, dyslexic individuals. Whether this thalamic disruption is specifically magnocellular is a subject of debate $[1,9,38]$ and is not particularly crucial to the present model. Similarly, Stein and Walsh propose that the magnocellular disruption further extends to the posterior parietal cortex and the cerebellum [1]. If true, this might well explain the visuospatial and motor symptoms observed in certain dyslexics.

In summary, not only do cognitive studies suggest that dyslexia is a specific phonological deficit associated with an optional sensorimotor syndrome, but also the neurobiological data seem perfectly compatible with this view and able to explain how this might be.

\section{Explaining heterogeneity within dyslexia}

One essential aspect of the proposed model is that it assumes that focal cortical abnormalities disrupt the development of the particular cognitive function(s) that would normally recruit those areas. Of course, there is not one single area assumed to be involved in phonological processing that would be disrupted in dyslexia. Rather, the phonological deficit of dyslexics is usually described as having three main components: poor phonological awareness (the ability to access and manipulate speech sounds consciously), slow lexical retrieval (evidenced in rapid serial naming tasks) and poor verbal short-term memory (as tested by digit span or non-word repetition). Each of these phonological skills in turn involves a whole network of cortical areas [39] (Figure 3c). Interestingly, the observed distribution of ectopias in dyslexic brains closely matches this network (compare Figure 3a with Figure 3c) as well as larger-scale structural anomalies (Figure 3b). But note that dyslexic brains vary in terms of both the number and the distribution of their ectopias. This suggests that there might be several ways to become dyslexic, depending on which subset of the phonological skills network is affected. This is indeed consistent with data showing that the different components of the phonological deficit vary partly independently and provide additive contributions to the reading disability [40]. In brief, variation in the symptoms of the phonological deficit might straightforwardly reflect variation in the distribution of the underlying cortical abnormalities.

\section{Generalizing to other developmental disorders}

From these premises, one might of course expect focal cortical abnormalities to sometimes arise outside the phonological network. By the same logic, one would then predict disruption to the development of the corresponding cognitive functions. This could provide a way to explain neurodevelopmental disorders other than dyslexia. For instance, ectopias in areas involved in syntax, morphology and/or the lexicon might engender the various manifestations of SLI. Similar abnormalities in the relevant areas might also explain developmental dyscalculia, developmental prosopagnosia, and at least some forms of autism, ADHD or dyspraxia.

It is particularly interesting to note that, as in dyslexia, a certain proportion of individuals affected by the 
disorders just mentioned present with just the same sort of sensorimotor impairments [3,41-45]. Animal models suggest a straightforward explanation for this: in both mice and rats, thalamic disruption occurred under the conjunction of high fœetal testosterone and ectopias or microgyri, whatever the location of these cortical abnormalities.

Therefore, the present model potentially explains not only the specific cognitive deficits characterizing many developmental disorders, but also the fact that these disorders are associated with an optional sensorimotor syndrome: in all disorders, additional hormonal conditions are the mediating factor to the sensorimotor symptoms.

\section{Explaining comorbidity between disorders}

Finally, the postulated structure-function relationship also provides an explanation for the typical comorbidity between different developmental disorders. Indeed, nothing restricts the distribution of cortical abnormalities within a given cognitive domain. If ectopias span, say, both the phonological and the syntactic systems, then the outcome would be a case of comorbid dyslexia and SLI. All other observed comorbidities can be explained accordingly.

\section{Generating new predictions}

One straightforward prediction of the model is that a whole class of domain-specific developmental disorders is characterized by similar focal brain anomalies, the differences between disorders reducing to differences in localization. Unfortunately, post-mortem work has been extremely limited past the original studies. Brain imaging studies of dyslexia, SLI and dyscalculia are certainly compatible with the present prediction [15] but, owing to resolution limitations, they are currently insufficient to test it seriously. Research on the neurobiology of autism and ADHD has shown rather different types of abnormalities $[46,47]$, but this does not exclude the possibility that certain cases of these disorders might be explained by focal anomalies of the same nature as dyslexia in relevant brain areas.

Because of the steroid hormonal mediation leading to the thalamic disruption, the model also predicts an increased prevalence of the sensorimotor syndrome in males (regardless of the actual sex ratio in dyslexia). More precisely, it predicts that the male:female ratio will be increased in the subpopulation with a sensorimotor syndrome, as compared with the subpopulation without it (in dyslexia as well as in other developmental disorders). Such predictions could be easily tested by carrying out post-hoc analyses on already existing datasets including reliable individual data on sensory and/or motor measures.

Another prediction of the model is that if one could measure the relevant hormonal conditions in human foetuses, and relate these measures to later outcome measures of sensorimotor functions, there would be significant correlations (more than with measures of each specific cognitive deficit). Only major longitudinal studies including all the relevant measures will be able to test this prediction. In the meantime, one might want to look for markers of fotal hormonal conditions that persist throughout development. A possible one is the ratio between the lengths of the second and fourth digits (2D:4D ratio), which is inversely correlated to fœtal testosterone levels [48] and significantly lower in autism than in the general population [49]. A recent replication further found that the $2 \mathrm{D}: 4 \mathrm{D}$ ratio was more specifically correlated with the performance of autistic children in visual and motor tasks [50], which is consistent with the model (applied to autism), although the evidence is still very preliminary and indirect.

The high heritability of developmental disorders such as dyslexia and SLI is consistent with the clear genetic origin of ectopias and related focal anomalies [51,52] (Y. Wang et al., unpublished). Furthermore, unless total cross-heritability between different disorders is shown, the model also predicts that the precise location of cortical anomalies is under genetic control. This is consistent with the fact that different strains of mutant mice have ectopias in different locations [29], but the exact mechanisms influencing their location are still unknown.

By contrast, fœetal hormonal conditions are more likely to be influenced by non-genetic factors. The model therefore predicts a lower heritability of the sensorimotor syndrome than of specific cognitive deficits, which is indeed the case (for auditory and visual versus phonological deficits) [53-55].

It is also notable that all the specific cognitive disorders under consideration here have a complex genetic aetiology, involving several regions on different chromosomes [56]. One way to understand this is to speculate that in these disorders, certain genes are general susceptibility factors for focal anomalies such as ectopias, whereas other genes control the precise location of such anomalies, for instance by generating molecular gradients interacting with ectopia susceptibility factors. This broadly predicts that the genes implicated in all these specific cognitive disorders will be partly shared (those acting as general susceptibility factors), and partly specific to each disorder (those determining specific brain locations). The more specific predictions are potentially testable using current mouse models.

\section{Concluding remarks}

The model outlined here is compatible with all the available cognitive and neurobiological evidence and, uniquely, also offers potential explanations for the association between specific cognitive developmental disorders and sensorimotor manifestations, heterogeneity within each disorder, and comorbidity between disorders.

Future research should now aim to uncover the precise links between specific genes, brain anomalies and cognitive deficits. To meet that challenge, research on developmental disorders will have to complete a methodological revolution that has only recently begun: the production and analysis of reliable individual data at all levels of description. Indeed, the present model suggests that several genetic, neurological and cognitive traits are consistently associated with dyslexia and other disorders, without actually explaining them. This implies that the usual studies focusing on group differences and correlations between measures are doomed to confuse core with 
associated deficits, and cause with correlation. The future belongs to longitudinal studies that will be able to trace causal pathways throughout development, across genetic, neurological and cognitive measures, and within each individual subject.

\section{Acknowledgements}

This work was supported by a Marie Curie fellowship of the European Community programme Quality of Life (QLGI-CT 1999-51305) and a research grant from the Fyssen Foundation. I thank Al Galaburda, Uta Frith, John Morton, Alfonso Caramazza and Tim Shallice for discussion and encouragement, and Jeff Lidz and Sarah White for comments on a previous version of this paper.

\section{References}

1 Stein, J.F. and Walsh, V. (1997) To see but not to read; the magnocellular theory of dyslexia. Trends Neurosci. 20, 147-152

2 Ramus, F. (2003) Developmental dyslexia: specific phonological deficit or general sensorimotor dysfunction? Curr. Opin. Neurobiol. $13,212-218$

3 Kadesjö, B. and Gillberg, C. (2001) The comorbidity of ADHD in the general population of Swedish school-age children. J. Child Psychol. Psychiatry 42, 487-492

4 McArthur, G.M. et al. (2000) On the 'specifics' of specific reading disability and specific language impairment. J. Child Psychol. Psychiatry 41, 869-874

5 Snowling, M.J. (2000) Dyslexia, Blackwell

6 Tallal, P. (1980) Auditory temporal perception, phonics, and reading disabilities in children. Brain Lang. 9, 182-198

7 Farmer, M.E. and Klein, R.M. (1995) The evidence for a temporal processing deficit linked to dyslexia: A review. Psychon. Bull. Rev. 2, 460-493

8 Lovegrove, W.J. et al. (1980) Specific reading disability: differences in contrast sensitivity as a function of spatial frequency. Science 210, $439-440$

9 Livingstone, M.S. et al. (1991) Physiological and anatomical evidence for a magnocellular defect in developmental dyslexia. Proc. Natl. Acad. Sci. U. S. A. 88, 7943-7947

10 Nicolson, R.I. and Fawcett, A.J. (1990) Automaticity: a new framework for dyslexia research? Cognition 35, 159-182

11 Nicolson, R.I. et al. (2001) Dyslexia, development and the cerebellum. Trends Neurosci. 24, 515-516

12 Ramus, F. et al. (2003) Theories of developmental dyslexia: Insights from a multiple case study of dyslexic adults. Brain 126, 841-865

13 Rosen, S. (2003) Auditory processing in dyslexia and specific language impairment: Is there a deficit? What is its nature? Does it explain anything? J. Phonetics 31, 509-527

14 Habib, M. (2000) The neurological basis of developmental dyslexia: an overview and working hypothesis. Brain 123, 2373-2399

15 Eckert, M. (2004) Neuroanatomical markers for dyslexia: a review of dyslexia structural imaging studies. Neuroscientist 10,362-371

16 Galaburda, A.M. and Kemper, T.L. (1979) Cytoarchitectonic abnormalities in developmental dyslexia: a case study. Ann. Neurol. 6, 94-100

17 Galaburda, A.M. et al. (1985) Developmental dyslexia: four consecutive patients with cortical anomalies. Ann. Neurol. 18, 222-233

18 Humphreys, P. et al. (1990) Developmental dyslexia in women: neuropathological findings in three patients. Ann. Neurol. 28, 727-738

19 Kaufmann, W.E. and Galaburda, A.M. (1989) Cerebrocortical microdysgenesis in neurologically normal subjects: a histopathologic study. Neurology 39, 238-244

20 Galaburda, A.M. et al. (1994) Evidence for aberrant auditory anatomy in developmental dyslexia. Proc. Natl. Acad. Sci. U. S. A. 91, 8010-8013

21 Galaburda, A.M. (1999) Developmental dyslexia: A multilevel syndrome. Dyslexia 5, 183-191

22 Herman, A.E. et al. (1997) Cerebral microgyria, thalamic cell size and auditory temporal processing in male and female rats. Cereb. Cortex 7 , 453-464

23 Peiffer, A.M. et al. (2002) Rapid auditory processing and MGN morphology in microgyric rats reared in varied acoustic environments. Brain Res. Dev. Brain Res. 138, 187-193
24 Fitch, R.H. et al. (1994) Induced microgyria and auditory temporal processing in rats: a model for language impairment? Cereb. Cortex 4 , 260-270

25 Peiffer, A.M. et al. (2001) Impaired detection of variable duration embedded tones in ectopic NZB/BINJ mice. NeuroReport 12, 2875-2879

26 Fitch, R.H. et al. (1997) Effects of sex and MK-801 on auditoryprocessing deficits associated with developmental microgyric lesions in rats. Behav. Neurosci. 111, 404-412

27 Peiffer, A.M. et al. (2002) Sex differences in rapid auditory processing deficits in ectopic BXSB/MpJ mice. NeuroReport 13, 2277-2280

28 Rosen, G.D. et al. (1999) Sex differences in the effects of early neocortical injury on neuronal size distribution of the medial geniculate nucleus in the rat are mediated by perinatal gonadal steroids. Cereb. Cortex 9, 27-34

29 Denenberg, V.H. et al. (1991) Spatial learning, discrimination learning, paw preference and neocortical ectopias in two autoimmune strains of mice. Brain Res. 562, 98-104

30 Schrott, L.M. et al. (1992) Environmental enrichment, neocortical ectopias, and behavior in the autoimmune NZB mouse. Brain Res. Dev. Brain Res. 67, 85-93

31 Balogh, S.A. et al. (1998) Effects of neocortical ectopias upon the acquisition and retention of a non-spatial reference memory task in BXSB mice. Brain Res. Dev. Brain Res. 111, 291-293

32 Rosen, G.D. et al. (1995) Behavioral consequences of neonatal injury of the neocortex. Brain Res. 681, 177-189

33 Boehm, G.W. et al. (1996) Neocortical ectopias in BXSB mice: effects upon reference and working memory systems. Cereb. Cortex 6, 696-700

34 Waters, N.S. et al. (1997) Effects of cortical ectopias on spatial delayedmatching-to-sample performance in BXSB mice. Behav. Brain Res. 84, 23-29

35 Hyde, L.A. et al. (2000) Working memory deficits in BXSB mice with neocortical ectopias. Physiol. Behav. 70, 1-5

36 Hyde, L.A. et al. (2001) Effects of ectopias and their cortical location on several measures of learning in BXSB mice. Dev. Psychobiol. 39, 286-300

37 Hyde, L.A. et al. (2002) Spatial and nonspatial Morris maze learning: impaired behavioral flexibility in mice with ectopias located in the prefrontal cortex. Behav. Brain Res. 133, 247-259

38 Skottun, B.C. (1997) The magnocellular deficit theory of dyslexia. Trends Neurosci. 20, 397-398

39 Turkeltaub, P.E. et al. (2003) Development of neural mechanisms for reading. Nat. Neurosci. $6,767-773$

40 Wolf, M. et al. (2002) The second deficit: An investigation of the independence of phonological and naming-speed deficits in developmental dyslexia. Reading Writing 151, 43-72

41 McArthur, G.M. and Bishop, D.V.M. (2001) Auditory perceptual processing in people with reading and oral language impairments: Current issues and recommendations. Dyslexia 7, 150-170

42 Hill, E.L. (2001) Non-specific nature of specific language impairment: a review of the literature with regard to concomitant motor impairments. Int. J. Lang. Commun. Disord. 36, 149-171

43 Milne, E. et al. (2002) High motion coherence thresholds in children with autism. J. Child Psychol. Psychiatry 43, 255-263

44 O'Brien, J. et al. (2002) Form and motion coherence processing in dyspraxia: evidence of a global spatial processing deficit. NeuroReport 13, 1399-1402

45 Duchaine, B.C. (2000) Developmental prosopagnosia with normal configural processing. NeuroReport 11, 79-83

46 Bailey, A. et al. (1998) A clinicopathological study of autism. Brain 121, 889-905

47 Castellanos, F.X. et al. (2002) Developmental trajectories of brain volume abnormalities in children and adolescents with attentiondeficit/hyperactivity disorder. JAMA 288, 1740-1748

48 Manning, J.T. et al. (1998) The ratio of 2 nd to 4th digit length: a predictor of sperm numbers and concentrations of testosterone, luteinizing hormone and oestrogen. Hum. Reprod. 13, 3000-3004

49 Manning, J.T. et al. (2001) The 2nd to 4th digit ratio and autism. Dev. Med. Child Neurol. 43, 160-164

50 Milne, E. et al. Motion and form coherence detection in autistic spectrum disorder: relationship to motor control and 2:4 digit ratio. J. Autism Dev. Disord. (in press) 
51 Sherman, G.F. et al. (1990) Brain abnormalities in immune defective mice. Brain Res. 532, 25-33

52 Sherman, G.F. et al. (1994) A genetic analysis of neocortical ectopias in New Zealand black autoimmune mice. NeuroReport 5, 721-724

53 Bishop, D.V. et al. (1999) Different origin of auditory and phonological processing problems in children with language impairment: evidence from a twin study. J. Speech Lang. Hear. Res. 42, 155-168
54 Davis, C.J. et al. (2001) Etiology of reading difficulties and rapid naming: the Colorado twin study of reading disability. Behav. Genet. 31, 625-635

55 Olson, R. and Datta, H. (2002) Visual-temporal processing in readingdisabled and normal twins. Reading Writing 15, 127-149

56 Fisher, S.E. et al. (2003) Deciphering the genetic basis of speech and language disorders. Annu. Rev. Neurosci. 26, 57-80

\section{Articles of interest in Current Opinion journals}

Regulation of exocytosis in neurons and neuroendocrine cells Seong An and David Zenisek

Current Opinion in Neurobiology 14, 522-530

Ephaptic interactions within a chemical synapse: hemichannel-mediated ephaptic inhibition in the retina

Maarten Kamermans and Iris Fahrenfort

Current Opinion in Neurobiology 14, 531-541

Spatial and temporal control of signaling through lipid rafts

Tamara Golub, Stefan Wacha and Pico Caroni

Current Opinion in Neurobiology 14, 542-550

Intrinsic neuronal regulation of axon and dendrite growth Jeffrey L. Goldberg

Current Opinion in Neurobiology 14, 551-557

Neurotrophin action on a rapid timescale

Yury Kovalchuk, Knut Holthoff and Arthur Konnerth

Current Opinion in Neurobiology 14, 558-563

Molecular motors in neuronal development, intracellular transport and diseases

Nobutaka Hirokawa and Reiko Takemura

Current Opinion in Neurobiology 14, 564-573

Binding proteins for mRNA localization and local translation, and their dysfunction in genetic neurological disease

Gary J. Bassell and Sofija Kelic

Current Opinion in Neurobiology 14, 574-581

Amyloid-beta precursor protein processing in neurodegeneration

Valérie Wilquet and Bart De Strooper

Current Opinion in Neurobiology 14, 582-588

Labelling neurons in vivo for morphological and functional studies

Paul Young and Guoping Feng

Current Opinion in Neurobiology 14, 642-646

Post-transcriptional gene silencing in neurons

Henry C. Zeringue and Martha Constantine-Paton

Current Opinion in Neurobiology 14, 654-659 